\title{
Study on the Role of Efflux Pump in Drug Resistance of Pseudomonas aeruginosa
}

\author{
R. Chavan*, A. De and S. Baveja \\ Department of Microbiology, L.T.M. Medical College, Mumbai, India \\ *Corresponding author
}

\begin{tabular}{|c|}
\hline Keywords \\
\hline $\begin{array}{l}\text { Efflux pump, } \\
\text { Resistance, } \\
\text { Pseudomonas } \\
\text { aeruginosa. }\end{array}$ \\
\hline Article Info \\
\hline $\begin{array}{l}\text { Accepted: } \\
15 \text { October } 2017 \\
\text { Available Online: } \\
10 \text { December } 2017\end{array}$ \\
\hline
\end{tabular}

\section{Introduction}

The 20th century was considered the antimicrobial era, whereas the 21 st century may well represent the post-antimicrobial era. The reason for this dramatic change, should it come to pass, is the development of bacterial resistance to antimicrobial agents. Multidrug resistant (MDR) and pan drug resistant (PDR) bacteria are on the rise, leaving clinicians with few or no therapeutic options ${ }^{1}$. An understanding of these important microbial resistance mechanisms will help the clinicians to identify circumstances in which resistance may be a problem, as well as evaluating the potential usefulness of an alternate antimicrobial agent against resistant microbes.

Pseudomonas aeruginosa is frequently implicated in healthcare associated infections (HAIs). Efflux pumps are transport proteins involved in the extrusion of toxic substrate, 
including all classes of clinically relevant antibiotics from within cells, into the external environment. In Gram negative organisms, efflux pump was first identified in Escherichia coli. Four different antibiotic efflux systems have been described in $P$. aeruginosa - MexAB-oprM, MexXY-oprM, MexCD-oprJ and MexEF-oprN. All classes of antibiotics except the polymyxins are susceptible to extrusion by one or more of these efflux systems. MexAB-oprM is responsible for extrusion of $\beta$-lactams, quinolones and a range of disinfectants. MexXY-oprM extrudes aminoglycosides. The third efflux operon MexEF-oprN confers resistance to quinolones, chloramphenicol and trimethoprim. $^{2}$

Use of efflux pump inhibitors improve and potentiate the activity of exported antibiotics. One known efflux pump inhibitor (EPI) in Pseudomonas aeruginosa is phenylalanine argynyl $\beta$-naphthylamide (PA $\beta N)$. It is apeptidomimetic compound, also called MC207,110. It is capable of significantly reducing fluoroquinolone resistance in $P$. aeruginosa. $^{2}$

Curcumin is a natural extract derived from rhizomes of the plant Curcuma longa (Zingiberaceae) grown in regions of India and South East Asia. Extensive research in this field has shown its activity as an antiinflammatory, antiviral, antioxidant and anticancer agent. It was observed that curcumin potentiated the effect of various antibiotics against Staphylococcus aureus and reduced the virulence in animal pathogenicity models. Effort is also being made to explore a natural analogue of the above drug curcumin, which may be biocompatible and safe for human consumption.

Therefore, this study was undertaken to detect minimum inhibiting concentration (MIC) of selected antibiotics; to detect reduction in
MIC by the use of an efflux pump inhibitor phenylalanine argynyl $\beta$-naphthylamide $(\mathrm{PA} \beta \mathrm{N})$ and to explore the possible efflux pump activity of a natural compound curcumin derived from curry spice turmeric (Curcuma longa) which may act as an efflux pump inhibitor.

\section{Materials and Methods}

A total of 126 laboratory confirmed non duplicate isolates of Pseudomonas aeruginosa from all clinical samples received in this tertiary care hospital in Mumbai were included in this study for the period of one year, i.e. from $1^{\text {st }}$ January to $31^{\text {st }}$ December 2014. All new born infants, geriatric patients and HIV positive patients were excluded. All isolates were identified by standard biochemical reactions ${ }^{3}$. Antibiotic susceptibility testing was done on Mueller Hinton Agar (MHA) plate by Minimum Inhibitory Concentration (MIC) by agar dilution method by using five antibiotics Ceftazidime, Ciprofloxacin, Gentamicin, Imipenem and Meropenem. Results were recorded as per Clinical Laboratory Standards Institute (CLSI) 2014 guidelines (M07-A9) ${ }^{4}$.

\section{Minimum inhibitory concentration (MIC) method $^{3}$}

Minimum inhibitory concentration (MIC) by agar dilution method was performed by using the same five antibiotics. Organisms were inoculated in 4ml Tryptic soy broth (HiMedia, Mumbai) and incubated overnight at $35^{\circ} \mathrm{C}$ and growth in broth was observed the next day.

The antibiotic was incorporated in MHA (Becton Dickinson and Co., Sparks, MD 21152, USA) plates by addition of appropriate concentrations of antibiotics in $20 \mathrm{ml}$ of molten MHA in a flask. Serial two-fold drug dilutions of respective antibacterial agents 
were made in dilution tubes. Each antibiotic was weighed and dissolved in $2 \mathrm{ml}$ of sterile triple distilled water in separate vials to make stock solutions of $4 \mathrm{mg} / \mathrm{ml}$. Then serial dilutions of each antibiotic solution ranging from $32 \mu \mathrm{g} / \mathrm{ml}$ to $0.25 \mu \mathrm{g} / \mathrm{ml}$ were made. Pseudomonas aeruginosa ATCC 27853 was used as positive control. Plates were incubated at $35^{\circ} \mathrm{C}$ for $16-20$ hours. MIC was recorded as the lowest concentration of antimicrobial agent that completely inhibited the growth. MIC was recorded as growth inhibition in antibiotic containing plate, when compared with plate without any antibiotic.

For ceftazidime, susceptible range was taken as $\leq 8 \mu \mathrm{g} / \mathrm{ml}$; Resistance range as $\geq 32 \mu \mathrm{g} / \mathrm{ml}$; Intermediate susceptible as $16 \mu \mathrm{g} / \mathrm{ml}$. For ciprofloxacin, susceptible range was taken as $\leq 1 \mu \mathrm{g} / \mathrm{ml}$; Resistance range as $\geq 4 \mu \mathrm{g} / \mathrm{ml}$; Intermediate susceptible as $2 \mu \mathrm{g} / \mathrm{ml}$. For gentamicin, susceptible range was taken as $\leq$ $4 \mu \mathrm{g} / \mathrm{ml}$; Resistance range as $\geq 16 \mu \mathrm{g} / \mathrm{ml}$; Intermediate susceptible as $8 \mu \mathrm{g} / \mathrm{ml}$. For imipenem, susceptible range was taken as $\leq$ $2 \mu \mathrm{g} / \mathrm{ml}$; Resistance range as $\geq 8 \mu \mathrm{g} / \mathrm{ml}$; Intermediate susceptible as $4 \mu \mathrm{g} / \mathrm{ml}$. For meropenem, susceptible range was taken as $\leq$ $2 \mu \mathrm{g} / \mathrm{ml}$; Resistance range as $\geq 8 \mu \mathrm{g} / \mathrm{ml}$; Intermediate susceptible as $4 \mu \mathrm{g} / \mathrm{ml}$. In this study, all intermediate susceptible strains were considered as susceptible.

Comparative MIC reduction after addition of Phenylalanine argynyl $\beta$-naphthylamide $(\mathbf{P A B N})^{5}$

Phenylalanine argynyl $\beta$-naphthylamide $(\mathrm{PA} \beta \mathrm{N})$, a broad spectrum efflux pump inhibitor, (Sigma Aldrich, USA) was dissolved in distilled water to prepare fixed concentrations of $50 \mu \mathrm{g} / \mathrm{mL}$. Stock solution of $10 \mathrm{mg} / \mathrm{ml}$ was prepared. Appropriate dilutions of each antimicrobial solution ranging from $32 \mu \mathrm{g} / \mathrm{ml}$ to $0.25 \mu \mathrm{g} / \mathrm{ml}$ were made and added to corresponding amount of molten MHA along with PA $\beta N$ separately. Uninoculated MHA plates and MHA with only PA $\beta N$ without antibiotics were included as growth controls in each experiment. The proportion of MIC reduction with PA $\beta \mathrm{N}$ was determined comparing the MIC of respective antimicrobials when tested alone.

Comparative MIC reduction study after addition of curcumin to each antibiotic solution $^{5}$

Curcumin (Hi-Media RM1449-54) was dissolved in Dimethyl Sulphoxide (DMSO) (Sigma Chemical, USA) and stock concentration of $10 \mathrm{mg} / \mathrm{ml}$ was stored at $20^{\circ} \mathrm{C}$. Final test concentrations were $20 \mu \mathrm{g} / \mathrm{ml}$, $30 \mu \mathrm{g} / \mathrm{ml}$ and $50 \mu \mathrm{g} / \mathrm{ml}$ for each sample. Appropriate dilution of each antimicrobial solution ranging from $32 \mu \mathrm{g} / \mathrm{ml}$ to $0.25 \mu \mathrm{g} / \mathrm{ml}$ are made and added to corresponding amount of molten MHA along with Curcumin in above concentrations separately. Uninoculated MHA plates and MHA with curcumin only without antibiotics were included as growth controls in each experiment. Proportion of MIC reduction with Curcumin was determined by comparing the MIC of antimicrobials when tested alone.

\section{Results and Discussion}

Majority of the isolates were from pus/wound swabs samples $(53.17 \%)$, followed by urine samples (19.84\%), as shown in Pie Diagram 1. By antibiotic susceptibility testing by MIC, most isolates were susceptible to Imipenem (59.52\%), followed by Meropenem (46.82\%) and Gentamicin (46.03\%). Resistance to ciprofloxacin was highest (71.43\%) (Bar Diagram2). Bar Diagram 3 shows MIC by agar dilution method with $\mathrm{Pa} \beta \mathrm{N}$. Susceptibility to imipenem, meropenem, ceftazidime and ciprofloxacin increased after addition of $\mathrm{PA} \beta \mathrm{N}$, but susceptibility to gentamicin remained same (Table 1). Total 
five isolates became susceptible to ceftazidime resistant strains and six to ciprofloxac in resistant strains after addition of PA $\beta N$. There was no change in total number of susceptible strains even after addition of PA $\beta \mathrm{N}$ to gentamicin. Total seven resistant strains became susceptible after addition of $\mathrm{PA} \beta \mathrm{N}$ to Imipenem and four resistant strains became susceptible after addition of PA $\beta \mathrm{N}$ to Meropenem (Table 1). MIC lowered significantly with addition of $\mathrm{PA} \beta \mathrm{N}$ in all antibiotics, except gentamicin in a total of $54(42.86 \%)$ strains (Table 2). Significant MIC reduction was highest in ciprofloxacin $(42.86 \%)$.

By using $20 \mu \mathrm{g} / \mathrm{ml}$ and $30 \mu \mathrm{g} / \mathrm{ml}$ concentrations of curcumin, no change was observed (Table 3). Using concentration of curcumin $50 \mu \mathrm{g} / \mathrm{ml}$, only one strain became susceptible to imipenem, two strains became susceptible to ceftazidime and meropenem, and three strains became susceptible to gentamicin, which were previously resistant to it (Table 4). Curcumin resulted in decrease in MIC significantly, which was seen maximum with meropenem, where a total of nine strains showed decrease in MIC after addition of curcumin. This was followed by imipenem, where six strains showed significant decrease in MIC. Table 5 shows the no. of strains in which MIC was lowered significantly after addition of PA $\beta N$ and curcumin.

Bacterial infectious diseases have been an important cause of morbidity and mortality in the history of mankind. The ability of bacteria to overcome antibacterial agents is increasing gradually.

Pseudomonas aeruginosa is a versatile human pathogen, particularly seen in critically ill or immunocompromised patients. ${ }^{6}$ They are responsible for morbidity, mortality and healthcare costs both in hospitals and in the community. $^{7}$ This 'superbug' is responsible for hospital associated infections (HAIs). Infections due to Pseudomonas aeruginosa is quite difficult to treat due to its rapidly developing inherent resistance mechanism against many antimicrobials and disinfectants. ${ }^{8}$ The difficulty in treatment of pseudomonas infections is due to its multidrug resistance (MDR) and exhibits resistance to most antimicrobial agents due to the expression of different mechanisms, overcoming their effects.

Pseudomonas aeruginosa is known for phenomenon of bacterial resistance, as practically all types of resistance mechanisms are seen. It exhibits resistance by virtue of expression of AmpC $\beta$-lactamase and efflux pumps, combined with a low permeability of the outer membrane. Its remarkable ability to acquire further resistance mechanisms to multiple groups of antimicrobial agents, including $\beta$-lactams, aminoglycosides and fluoroquinolones.

Efflux pumps in $P$. aeruginosa belong to Resistance Nodulation Division (RND) family. MexA-MexB-OprM, MexC-MexDOprJ, MexE-MexF-OprN and MexXMexY-OprM are common efflux pumps known in P.aeruginosa.,

This study was undertaken to know the role of efflux pump in drug resistance of Pseudomonas aeruginosa. For this, a known efflux pump inhibitor Phenylalanine argynyl $\beta$-naphthylamide $(\mathrm{PA} \beta \mathrm{N})$, a broadspectrum synthetic and toxic efflux pump inhibitor was used. Also, curcumin, a natural compound (a herbal extract) was also investigated for its possible role as an efflux pump inhibitor.

The present study of one year duration was undertaken to investigate the contribution of efflux pumps in drug resistance of 
P. aeruginosa prevailing amongst hospital isolates from patients admitted in different wards of a tertiary care hospital in Mumbai. In this study, $126 P$. aeruginosa strains were included, which were isolated from wound swabs/pus, endotracheal aspirates, sputum, urine, blood, etc.

Antibiotic susceptibility pattern of $P$. aeruginosa isolates by Minimum Inhibitory Concentration (MIC) by agar dilution method

Minimum Inhibitory Concentration (MIC) study was done for all $126 P$. aeruginosa isolates by using all the above five antibiotics by agar dilution method and susceptible, intermediate susceptible and resistant ranges taken were according to CLSI 2014 guidelines. All intermediate susceptible strains were considered as susceptible in this study. Maximum resistance was seen in Ciprofloxacin (71.43\%), followed by Ceftazidime and Gentamycin $(55.56 \%$ and $53.97 \%$ ) respectively. Resistance to Imipenem and Meropenem was $(40.48 \%$ and $53.17 \%$ respectively (Bar Diagram2).

In a study by Negi et al., resistance to ciprofloxacin was $57.64 \%$, followed by gentamicin $51.17 \%$, whereas resistance to ceftazidime and meropenem was $41.76 \%$ and $29.41 \%$ respectively by MIC. ${ }^{5}$ In a study by Shashikala et al., resistance to imipenem and meropenem was $10.9 \%$ each, resistance to both ciprofloxacin and gentamicin was 97\% among imipenem resistant $P$. aeruginosa by MIC. ${ }^{10}$ In another study by Ballal et al., $P$. aeruginosa isolates showed $0 \%$ resistance to imipenem, resistance to ciprofloxacin and gentamicin was $60 \%$ and $54 \%$ respectively, followed by ceftazidime $(44 \%)^{11}$.

In a study by Carmeli et al., from USA, 7\% strains were resistant to ceftazidime, $13 \%$ were resistant to imipenem and $21 \%$ were resistant to ciprofloxacin by $\mathrm{MIC}^{12}$, which is lower than the present study. In a study by Khan et al., from Pakistan, resistance pattern by MIC, for gentamycin was $67.8 \%$, which is much higher as compared to the present study and for ceftazidime, meropenem and imipenem it was $30.2 \%, 28 \%$ and $26.7 \%$ respectively ${ }^{13}$.

A study from Jakarta found resistance to both ceftazidime and gentamicin to be $68 \%$ and ciprofloxacin $44 \%$, by MIC, whereas the carbapenems showed good activity - only $20 \%$ and $25 \%$ resistance to imipenem and meropenem respectively ${ }^{14}$.

A surveillance study was conducted by Obritsch et al., over a 10-year period from 1993 to 2002 in patients admitted in ICUs in District of Columbia. They observed increasing resistance to all antipseudomonal agents, with the greatest increase involving ciprofloxacin (15 to $32 \%$ ), imipenem (15 to $23 \%$ ) and ceftazidime (15 to $19 \%)^{15}$.

This indicates that significant increase in resistance of $P$. aeruginosa isolates over period of time, which may compromise the ability to choose efficacious empirical regimens for treatment of this formidable pathogen in critically ill patients.

\section{MIC of $P$. aeruginosa with selected} antibiotics after addition of PA $\mathbf{P N}$

When MIC was performed after adding $\mathrm{PA} \beta \mathrm{N}$, it was found that five $(7.14 \%)$ strains which were previously resistant to ceftazidime, became susceptible to it (Table 1), indicating the mechanism of resistance in these strains to be due to efflux pumps. A study from Canada by Lamers et al., showed that $\mathrm{PA} \beta \mathrm{N}$ reduced the minimal inhibitory concentrations (MICs) of several $\beta$-lactam antibiotics against P.aeruginosa $a^{16}$. 
Table 1. Susceptibility pattern of $P$. aeruginosa by MIC with and without PAßN (n=126)

\begin{tabular}{|c|c|c|c|}
\hline Antibiotic & $\begin{array}{c}\text { Total no. of } \\
\text { susceptible } \\
\text { strains } \\
\text { No. (\%) }\end{array}$ & $\begin{array}{c}\text { Total no. of } \\
\text { strains } \\
\text { susceptible after } \\
\text { addition of } \\
\text { PapN } \\
\text { No. (\%) }\end{array}$ & $\begin{array}{c}\text { Total no. of } \\
\text { strains showing } \\
\text { resistance due to } \\
\text { efflux pump } \\
\text { No. (\%) }\end{array}$ \\
\hline Ceftazidime & $56(44.44 \%)$ & $61(48.41 \%)$ & $05(7.14 \%)$ \\
\hline Ciprofloxacin & $36(28.57 \%)$ & $42(33.33 \%)$ & $06(6.67 \%)$ \\
\hline Gentamicin & $58(46.03 \%)$ & $58(46.03 \%)$ & $00(0.00 \%)$ \\
\hline Imipenem & $75(59.53 \%)$ & $82(65.08 \%)$ & $07(13.73 \%)$ \\
\hline Meropenem & $59(46.83 \%)$ & $63(50 \%)$ & $04(5.97 \%)$ \\
\hline
\end{tabular}

Table 2. Strains in which MIC was lowered significantly after addition of PABN and Curcumin

\begin{tabular}{|l|c|c|}
\hline \multicolumn{1}{|c|}{ Antibiotic } & PAßN & Curcumin \\
\hline Ceftazidime $(\mathrm{n}=70)$ & $27(21.43 \%)$ & $04(3.17 \%)$ \\
\hline Ciprofloxacin $(\mathrm{n}=90)$ & $54(42.86 \%)$ & $02(1.59 \%)$ \\
\hline Gentamicin $(\mathrm{n}=68)$ & $04(3.17 \%)$ & $03(2.38 \%)$ \\
\hline Imipenem $(\mathrm{n}=51)$ & $15(11.90 \%)$ & $06(4.76 \%)$ \\
\hline Meropenem $(\mathrm{n}=67)$ & $13(10.31 \%)$ & $09(7.14 \%)$ \\
\hline
\end{tabular}


Table 3. MIC of Pseudomonas aeruginosa by agar dilution method using different concentrations of Curcumin $(\mathrm{n}=126)$

\begin{tabular}{|l|c|c|c|c|c|c|}
\hline \multirow{2}{*}{ Antibiotic } & \multicolumn{2}{|c|}{ Curcumin 20 $\boldsymbol{\mu g} / \mathbf{m l}$} & \multicolumn{2}{c|}{ Curcumin 30 $\boldsymbol{\mu g} / \mathbf{m l}$} & \multicolumn{2}{c|}{ Curcumin 50 $\boldsymbol{\mu g} / \mathbf{m l}$} \\
\cline { 2 - 7 } & $\mathrm{S}$ & $\mathrm{R}$ & $\mathrm{S}$ & $\mathrm{R}$ & $\mathrm{S}$ & $\mathrm{R}$ \\
\hline Ceftazidime & 54 & 72 & 54 & 72 & 56 & 70 \\
\hline Ciprofloxacin & 38 & 88 & 38 & 88 & 38 & 88 \\
\hline Gentamicin & 57 & 69 & 57 & 69 & 60 & 66 \\
\hline Imipenem & 75 & 51 & 75 & 51 & 76 & 50 \\
\hline Meropenem & 61 & 65 & 61 & 65 & 61 & 65 \\
\hline
\end{tabular}

Table 4. Comparison between sensitivity of $P$. aeruginosa strains with and without addition of $50 \mu \mathrm{g} / \mathrm{ml}$ Curcumin

\begin{tabular}{|l|c|c|}
\hline Antibiotic & $\begin{array}{c}\text { Total no. of susceptible } \\
\text { strains } \\
\text { No. (\%) }\end{array}$ & $\begin{array}{c}\text { Total no. of susceptible } \\
\text { strains after addition of 50 } \\
\boldsymbol{\mu g} / \mathbf{m l} \text { Curcumin } \\
\text { No. (\%) }\end{array}$ \\
\hline Ceftazidime & $56(44.44 \%)$ & $56(44.44 \%)$ \\
\hline Ciprofloxacin & $36(28.57 \%)$ & $38(30.16 \%)$ \\
\hline Gentamycin & $58(46.03 \%)$ & $60(47.62 \%)$ \\
\hline Imipenem & $75(59.52 \%)$ & $76(60.32)$ \\
\hline Meropenem & $59(46.83 \%)$ & $61(48.41 \%)$ \\
\hline
\end{tabular}


Table 5. Comparison of total number of $P$. aeruginosa strains which became susceptible after addition of PA $\mathrm{PN}$ and curcumin

\begin{tabular}{|l|c|c|}
\hline \multicolumn{1}{|c|}{ Antibiotic } & $\begin{array}{c}\text { Susceptible strains } \\
\text { after addition of } \\
\text { PAßN }\end{array}$ & $\begin{array}{c}\text { Susceptible } \\
\text { strains after } \\
\text { addition of } \\
\text { Curcumin }\end{array}$ \\
\hline Ceftazidime $(\mathrm{n}=70)$ & $05(7.14 \%)$ & $00(0.00 \%)$ \\
\hline Ciprofloxacin $(\mathrm{n}=90)$ & $06(6.67 \%)$ & $02(2.22 \%)$ \\
\hline Gentamicin $(\mathrm{n}=68)$ & $00(0.00 \%)$ & $02(2.94 \%)$ \\
\hline Imipenem $(\mathrm{n}=51)$ & $07(13.73 \%)$ & $01(1.96 \%)$ \\
\hline Meropenem $(\mathrm{n}=67)$ & $04(5.97 \%)$ & $02(2.99 \%)$ \\
\hline
\end{tabular}

Pie diagram 1

\section{SAMPLE WISE DISTRIBUTION OF 126 ISOLATES}

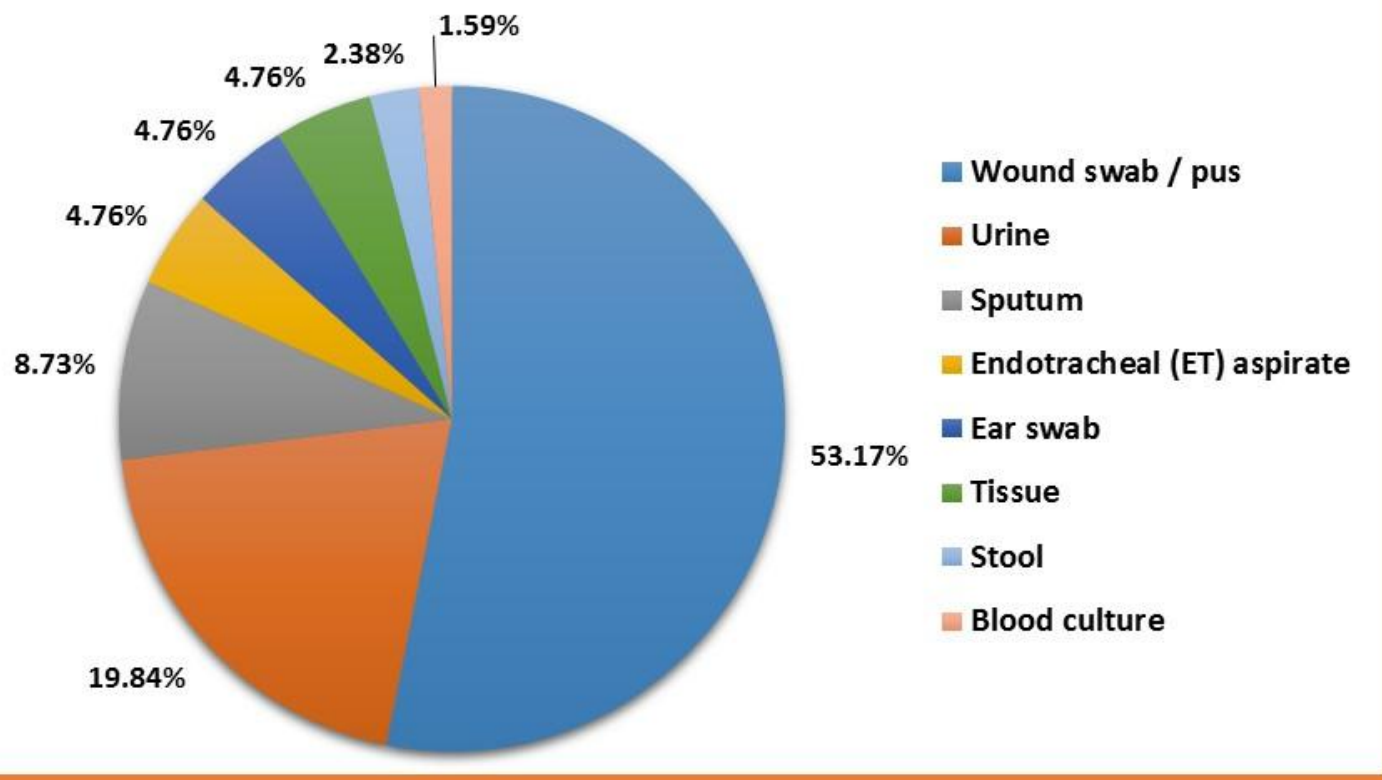


Bar diagram 1

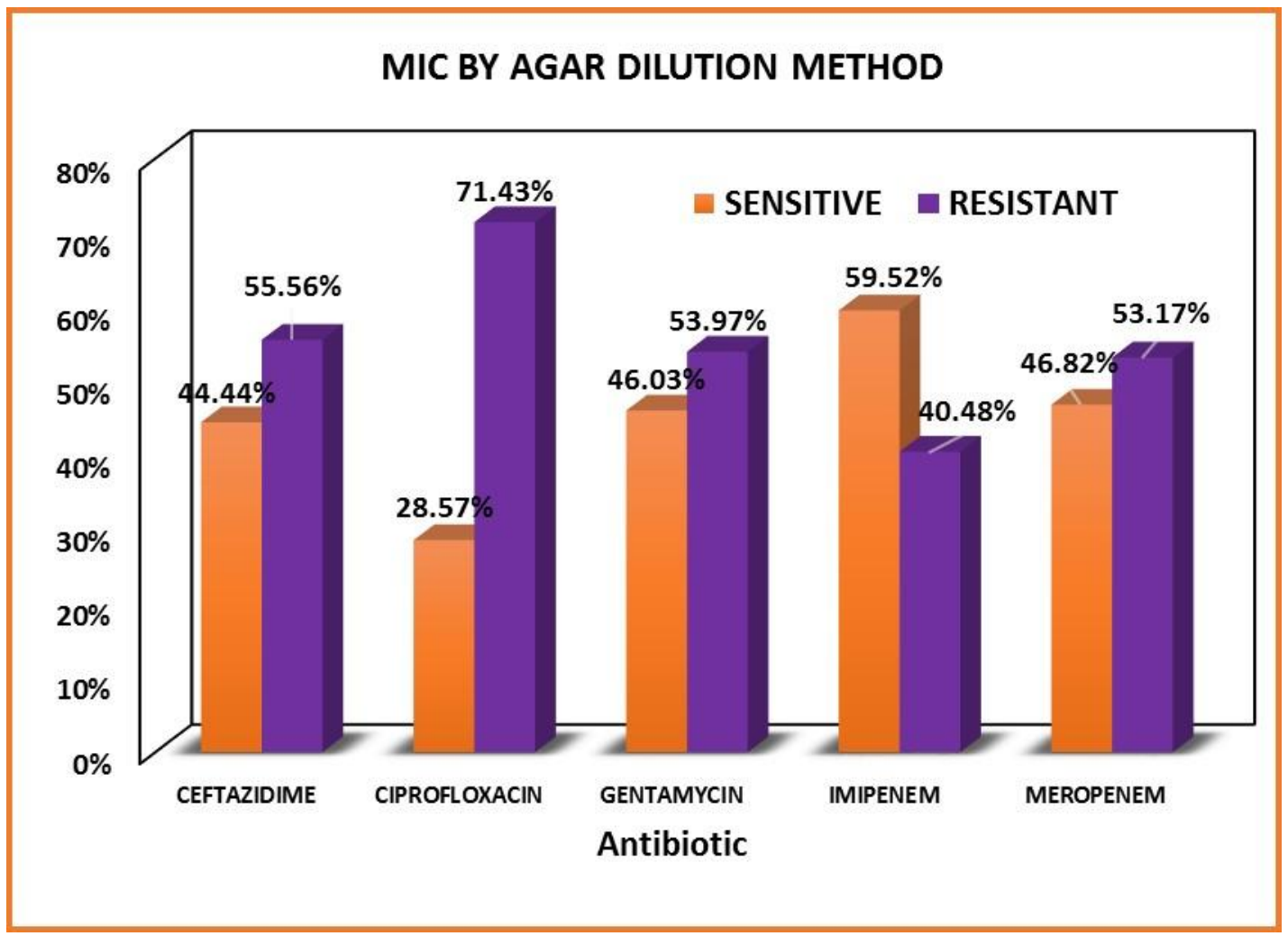

Bar Diagram 2

\section{MIC by agar dilution method with PABN}

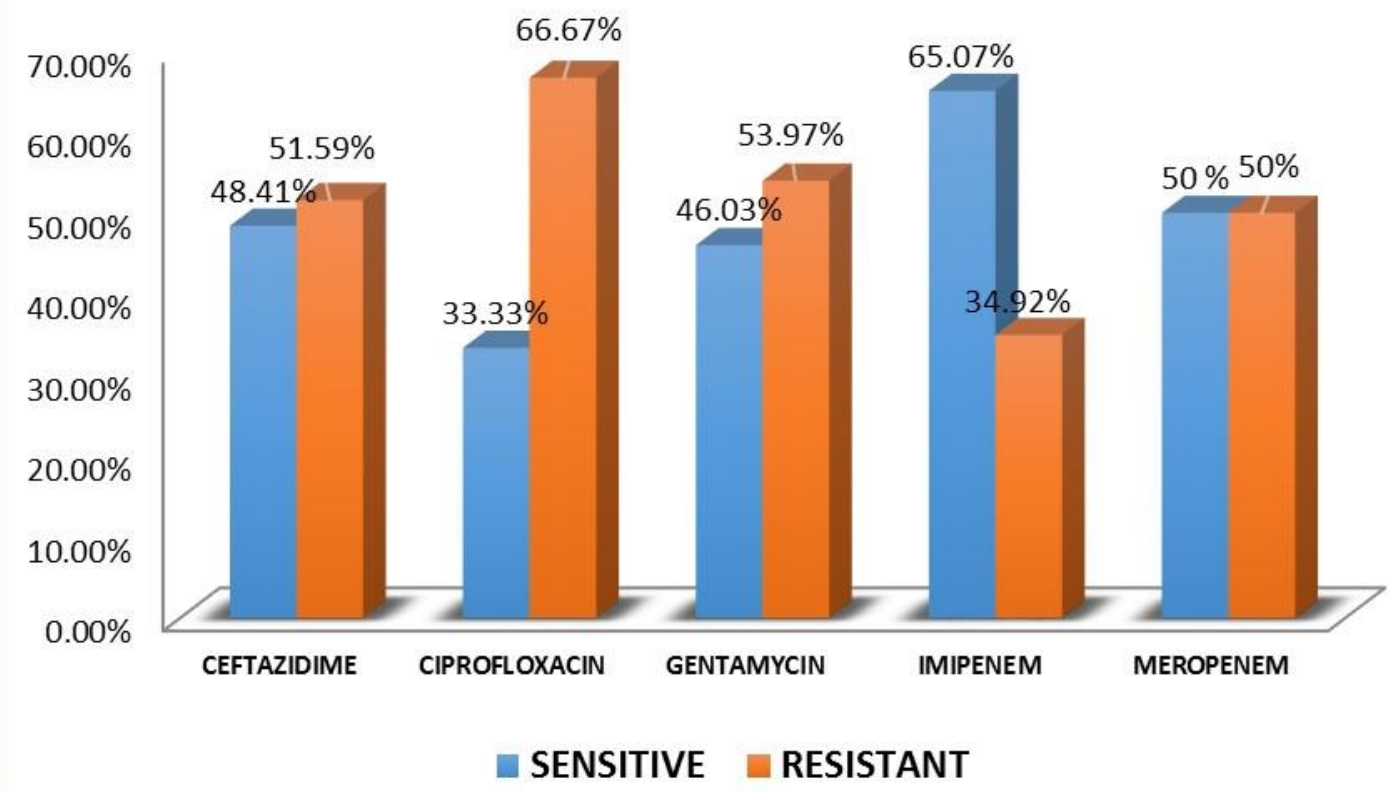


In the present study, MIC for ceftazidime lowered significantly in $27(21.43 \%)$ strains (Table 2). In a study by Negi et al., from North India, $21.12 \%$ strains showed resistance to ceftazidime due to efflux pumps ${ }^{5}$.

A total of six $(6.67 \%)$ strains which were previously resistant to ciprofloxacin, became susceptible after addition of PA $\beta N$ (Table 1). This is close to the findings of an Indian study, where they found $9.18 \%$ strains resistant due to efflux pumps. ${ }^{5}$ Another study from Belgium, showed decrease in MIC of a fluroquinolone (norfloxacin), where $57.14 \%$ strains became susceptible ${ }^{17}$.

In the present study, MIC significantly lowered after addition of PA $\beta \mathrm{N}$ in a total of $54(42.86 \%)$ strains (Table 2$)$. In a study from Egypt, PA $\beta \mathrm{N}$ resulted in significant reduction in the MIC of ciprofloxacin in $71.4 \%$ isolates ${ }^{18}$.A significant reduction was also seen in MIC to levofloxacin in a study by Lomavskya et al., ${ }^{19}$.

In case of gentamicin, there was no reduction in resistant strains after addition of PA $\beta \mathrm{N}$ (Table 1), which is in contrast to the study by Negi et al., ${ }^{5}$, who showed $14.9 \%$ strains showed resistance to gentamicin due to efflux pumps. In a study by Mesaros et al., two out of seven strains became susceptible to gentamicin after addition of $P A \beta N^{17}$. However, in the present study, in four $(3.17 \%)$ strains, MIC for gentamicin lowered significantly after addition of PA $\beta \mathrm{N}$ (Table 2).

A total of seven (13.73\%) strains which were previously resistant, became sensitive to imipenem after addition of PA $\beta \mathrm{N}$ (Table 1). In $15(11.90 \%)$ strains, MIC lowered significantly after addition of PA $\beta \mathrm{N}$ (Table 2 ). In a similar study at Belgium, only one strain of imipenem became susceptible and three strains showed decrease in $\mathrm{MIC}^{17}$.
A total of four $(5.97 \%)$ strains which were previously resistant became sensitive to meropenem after addition of PA $\beta \mathrm{N}$ (Table 1), indicating the resistance due to efflux pumps. In the present study, in $13(10.31 \%)$ strains, MIC for meropenem lowered significantly after addition of PA $\beta \mathrm{N}$ (Table 2).

However, in another study from India ${ }^{5}, 26 \%$ strains showed resistance due to efflux pump mechanism, which is much higher as compared to the present study.

\section{MIC of $P$. aeruginosa with selected} antibiotics after addition of curcumin

In the present study, curcumin, which is a natural compound, i.e. an herbal extract, was also studied for its possible role as an efflux pump inhibitor. When it was used in concentration of $20 \mu \mathrm{g} / \mathrm{ml}$ and $30 \mu \mathrm{g} / \mathrm{ml}$, no significant change in total number of susceptible strains were observed. Change was observed at concentration of $50 \mu \mathrm{g} / \mathrm{ml}$ only (Table 3 ).

Two previously resistant strains became susceptible after addition of curcumin to ciprofloxacin, gentamicin and meropenem individually. When curcumin was added to imipenem, only one previously resistant strain became susceptible to it. No change in total susceptible strains was observed after addition of curcumin to ceftazidime (Table 4).

This finding is similar to the study by Negi et $a l .{ }^{5}$ who found that two strains which were resistant previously became susceptible to ciprofloxacin and gentamicin each, after addition of curcumin.

After adding curcumin to meropenem, five resistant strains became susceptible. However, in their study six resistant strains became susceptible to ceftazidime, which is in contrast to the present study. 


\section{Comparison of PA $\beta N$ and curcumin}

When we compared the efflux pump inhibiting activity of $\mathrm{PA} \beta \mathrm{N}$ and curcumin, it was found that 22 strains became susceptible which were previously resistant after addition of PA $\beta N$. After addition of curcumin, only seven strains which were previously resistant became susceptible (Table 5). Therefore, in the present study, it was observed that PA $\beta \mathrm{N}$ is a better efflux pump inhibitor as compared to curcumin but the former has toxic properties whereas the latter is non-toxic herbal product, safe for human consumption.

Amongst 47 multi drug resistant strains, eleven $(23.4 \%)$ strains became susceptible after addition of PA $\beta \mathrm{N}$ but only five $(10.63 \%)$ strains became susceptible after addition of curcumin. In the study by Negi et al., ${ }^{5} 30 \%$ MDR isolates of $P$. aeruginosa showed decrease in MIC to susceptible level after addition of curcumin, which is much higher as compared to the present study.

In this study, overall resistance due to efflux pump in multidrug resistant $P$. aeruginosa was demonstrated in $14.89 \%$ (7/47) patients. Henrichfreise et al., from Germany demonstrated efflux pumps in $40.9 \%$ amongst multidrug resistant $P$. aeruginosa, which is much higher than the present study ${ }^{20}$.

Although synthetic efflux pump inhibitor (EPI) $\mathrm{PA} \beta \mathrm{N}$ is better than natural extract curcumin in inhibiting the resistant strains but keeping in mind their toxic potential and harmful effects, search for a natural homologue other than curcumin, which can mimic their action can never be ignored.

It might be acting either as drug analogue, hence becoming a substrate for efflux channels or it may inhibit the expression of different genes responsible for efflux pump mechanism.
The present study indicates the presence of efflux pump mediated drug resistance among clinical isolates of $P$. aeruginosa against one or more antimicrobials employed. Overall resistance due to efflux pump was seen in $14.89 \%$ MDR strains of $P$. aeruginosa. This study indicates both $\mathrm{PA} \beta \mathrm{N}$ and curcumin as potential EPIs, with PA $\beta \mathrm{N}$ being a better one but further confirmation is required by observing the molecular mechanisms.

Considering the seriousness of prevailing and emerging drug resistance in Pseudomonas aeruginosa, extensive research is needed for finding out some suitable alternatives, e.g. a synthetic EPI with less toxic effects, or a better natural extract like curcumin.

\section{References}

1. Struelens MJ. The Epidemiology of Antimicrobial resistance in hospital acquired infections: Problems and possible solutions. BMJ 1998; 317(7159): 652-4.

2. Askoura M, Mattawa W, Abujamel T, and Taher I. Efflux pump inhibitors (EPIs) as new antimicrobial agents against Pseudomonas aeruginosa. Libyan J Med 2011, 6: 5870.

3. Collee JG, Miles RS, Watt B, Marr W, and Amyes G B. Tests for the identification of bacteria. Specimen collection, Culture containers and media. Laboratory Control of Antimicrobial Therapy. Mackie and McCartney's Practical Medical Microbiology.14th Ed. Eds. Collee JG, Fraser AG, Marmion BP, Simmons A. Churchill Livingstone, Elsevier imprint. 2006; pp95113, 131-49, 151-66.

4. Performance standards for Antimicrobial susceptibility testing; Twenty Fourth informational supplement, Clinical and Laboratory Standards Institute. M100-S24. 2014; pp58-59, 176, 184.

5. Negi N., Prakash P., Gupta M., and Mohapatra T. Possible Role of Curcumin as an Efflux Pump Inhibitor in Multi Drug Resistant Clinical Isolates of Pseudomonas aeruginosa. J Clin Diagn Res 2014; 8(10): DC04-07. 
6. Christopher O, and Mathew P. Infections due to Pseudomonas species and related organisms. In Harrison's Principles of Internal Medicine. $16^{\text {th }}$ Ed. Eds. Kasper DL, Braunwald, Fauci, Hauser, Longo, Jameson. 2005; pp889-96.

7. Chander A, and Raza MS. Antimicrobial Susceptibility Patterns of Pseudomonas aeruginosa. Clinical Isolates - at a Tertiary Care Hospital in Kathmandu, Nepal. Asian J Pharm Clin Res2013; 6 (S3):235-8.

8. Lambert PA. Mechanisms of antibiotic resistance in Pseudomonas aeruginosa. $J R$ Soc Med 2002; 95(41): 22-6.

9. Tegos GP, Haynes M, Strouse JJ, Khan MT, Bologa CG, and Oprea TI et al., Microbial Efflux Pump Inhibition: Tactics and Strategies. Curr Pharm Des 2011; 17(13): 1291-1302.

10. Shashikala, Kanungo R, Srinivasan S, and Sheela Devi. Emerging Resistance to Carbapenems in hospital acquired Pseudomonas infection: A cause for concern. Indian J Pharmacol 2006; 38(4): 287-8.

11. Ballal M, ShenoyV P, Shivananda PG, and Bairy I. Honey as an Antimicrobial agent against Pseudomonas aeruginosa isolated from infected wounds. $J$ Glob Infect Dis 2012; 4(2): 102-5.

12. Carmeli Y, Troillet N, Eliopoulos G M, and Samore M H. Emergence of antibioticresistant Pseudomonas aeruginosa: comparison of risks associated with different antipseudomonal agents. Antimicrob Agents Chemother 1999; 43(6): 1379-82.

13. Khan JA, Iqbal Z, Rahman S U, Farzana K, and Khan A. Prevalence and resistance pattern of Pseudomonas aeruginosa against various antibiotics. Pak J Pharm Sci 2008; 21(3): 311-5.

14. Moehario L H, Hartono T S, Wardoyo E H, and Tjoa E. Trend of antibiotics susceptibility of multidrugs resistance Pseudomonas aeruginosa in Jakarta and surrounding areas from 2004 to 2010. African J Microbiol Res 2012; 6(9): 2222-9.

15. Obritsch M D, Fish D N, MacLaren R, and Jung R. National surveillance of antimicrobial resistance in Pseudomonas aeruginosa isolates obtained from intensive care unit patients from 1993 to 2002. Antimicrob Agents Chemother 2004; 48(12): 4606- 7.

16. Lamers RP, Cavallari JF, and Burrows LL. The Efflux Inhibitor Phenylalanine-Arginine Beta-Naphthylamide $(\mathrm{PA} \beta \mathrm{N})$ Permeabilizes the Outer Membrane of Gram-Negative Bacteria. PLoS ONE 2013; 8(3): e60666.

17. Mesaros N, Glupczynski Y, Avrain L, Caceres N E, Tulkens P M, and VanBambeke F. A combined phenotypic and genotypic method for the detection of Mex efflux pumps in Pseudomonas aeruginosa. $J$ Antimicrob Chemoth, 2007; 59: 378-86.

18. El-Said E E, Ali S A, Zakaria D M, Tawfiek A M, Abd E 1 Haliem N, Ali H A, and Ebrahem H A. Efflux Pump Contribution to Multidrug Resistance in Pseudomonas aeruginosa and the Effect of Using an Efflux Pump Inhibitor on Ciprofloxacin Resistance. Egyptian J Med Microbiol 2012; 21(2): 91104.

19. Lomovskaya O, Warren M S, Lee A, Galazzo J, Fronko R, and Lee $\mathrm{M}$ et al., Identification and Characterization of Inhibitors of Multidrug Resistance Efflux Pumps in Pseudomonas aeruginosa: Novel Agents for Combination Therapy. Antimicrob Agents Chemother, 2001; Jan; 45(1):105-16.

20. Henrichfreise B, Wiegand I, Pfister W, and Wiedemann B. Resistance Mechanisms of Multiresistant Pseudomonas aeruginosa Strains from Germany and Correlation with Hypermutation. Antimicrob Agents Chemother, 2007; 51:4062-70.

\section{How to cite this article:}

Chavan, R., A. De and Baveja, S. 2017. Study on the Role of Efflux Pump in Drug Resistance of Pseudomonas aeruginosa. Int.J.Curr.Microbiol.App.Sci. 6(12): 1944-1955. doi: https://doi.org/10.20546/ijcmas.2017.612.222 